\title{
Re-interpretation of the ERMINE-V experiment validation of fission product integral cross section in the fast energy range
}

\author{
Paul Ros ${ }^{\mathrm{a}}$, Pierre Leconte, Patrick Blaise, and Laurent Naymeh \\ CEA, DEN, DER, Cadarache, 13108 Saint-Paul-lez-Durance, France
}

\begin{abstract}
The current knowledge of nuclear data in the fast neutron energy range is not as good as in the thermal range, resulting in larger propagated uncertainties in integral quantities such as critical masses or reactivity effects. This situation makes it difficult to get the full benefit from recent advances in modeling and simulation. Zero power facilities such as the French ZPR MINERVE have already demonstrated that they can contribute to significantly reduce those uncertainties thanks to dedicated experiments. Historically, MINERVE has been mainly dedicated to thermal spectrum studies. However, experiments involving fast-thermal coupled cores were also performed in MINERVE as part of the ERMINE program, in order to improve nuclear data in fast spectra for the two French SFRs: PHENIX and SUPERPHENIX. Some of those experiments have been recently revisited. In particular, a full characterization of ZONA-1 and ZONA-3, two different cores loaded in the ERMINE V campaign, has been done, with much attention paid to possible sources of errors. It includes detailed geometric descriptions, energy profiles of the direct and adjoint fluxes and spectral indices obtained thanks to Monte Carlo calculations and compared to a reference fast core configuration. Sample oscillation experiments of separated fission products such as ${ }^{103} \mathrm{Rh}$ or ${ }^{99} \mathrm{Tc}$, which were part of the ERMINE $\mathrm{V}$ program, have been simulated using recently-developed options in the TRIPOLI-4 code and compared to the experimental values. The present paper describes the corresponding results. The findings motivate indepth studies for designing optimized coupled-core conditions in ZEPHYR, a new ZPR which will replace MINERVE and will provide integral data to meet the needs of Gen-III and Gen-IV reactors.
\end{abstract}

\section{Introduction}

Zero Power Reactors (ZPR) stand as a key tool for the development and the safe operation of nuclear reactors. From the first years of reactor physics, they have provided crucial information within two main frames: validation of neutronics tools and improvement of nuclear data.

Due to the industrial development of LWR, nuclear data in the thermal range have been widely studied through several dedicated programs worldwide [1]. Nevertheless, and despite numerous dedicated programs in fast ZPRs, cross-sections in the fast neutron energy range does not benefit from the same accuracy. It involves both the actual cross-section data of some actinides and fission products, as well as their associated uncertainties [2,3].

In France, a choice has been made a few years ago of performing further research on Fast Reactors through the ASTRID project [4]. It leads us to use, re-interpret and improve the work done by the pioneers during the 1970's and 1980's for PHENIX and SUPERPHENIX.

During those years, oscillations in fast-thermal coupled cores were used to determine both integral cross-sections of fission products and the loss of reactivity thanks to irradiated fuel samples in Fast Reactors [5].

This paper is dedicated to measurement of integral cross sections in the fast energy range through fast-thermal coupled experiments in the French CEA MINERVE facility, and their recent interpretation. It begins with a demonstration of pertinence of fast-thermal coupled

a e-mail: paul.ros@cea.fr experiments using modern calculation tools. For this purpose, we detail the ERMINE-V campaign conducted between 1976 and 1980 in MINERVE, and we focus on two cores called ZONA1 and ZONA3. Then, we present a quantitative re-interpretation of the oscillation experiments of separated fission products that occurred to improve $a$ posteriori ND accuracy.

\section{Principles of fast-thermal coupled cores}

ZPR facilities have been used within a wide range of spectra because their capacity for highly precise measurements is rare. It relies on several experimental techniques including oscillations. ZPR are using a two zones configuration, such as in MINERVE [1], RB [6] or RRR [7]. A measurement zone in the center has the targeted spectrum, representative of an industrial application. At the periphery, a driver zone enables the criticality by producing the main quantity of neutrons. This driver zone is usually a thermal spectrum zone.

The difficulty relies in the contrast between the two zones: between the thermal driver zone and the fast zone, an adaptation zone is designed.

Such cores allow a high reduction of the critical mass thanks to the reactivity of the thermal driver zone. Nonetheless, such an adaptation brings doubts on the quality of the spectrum in the center: do the neutronics conditions in the center - forward and adjoint flux - keep some spectral characteristics of the thermal driver zone?

(C) The Authors, published by EDP Sciences. This is an Open Access article distributed under the terms of the Creative Commons Attribution License 4.0 (http://creativecommons.org/licenses/by/4.0/). 


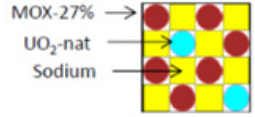

Figure 1. ZONA1-cell.

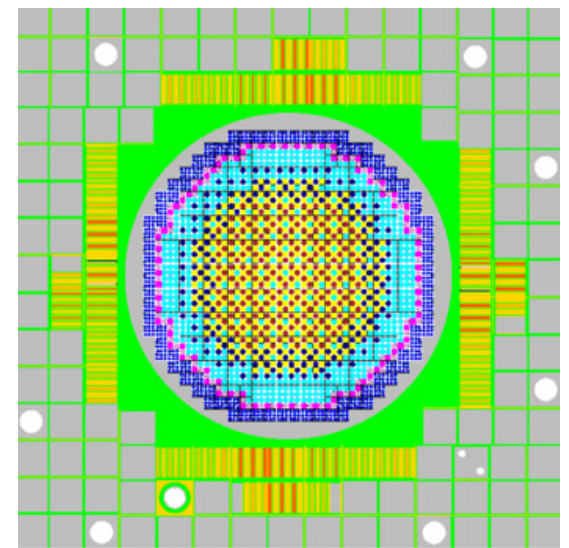

Figure 2. ZONA1 core.

\section{The ERMINE-V campaign}

\subsection{Generalities}

The ERMINE campaigns stand as a special program in the history of the MINERVE reactor. Indeed, it represents the only fast spectrum experiments ever been conducted in this facility. However, they took place between 1966 (ERMINE I) and 1985 (ERMINE VI) in order to support the design and operation of both PHENIX and SUPERPHENIX. The measurements covered several needs associated to fast reactors: Doppler effect, capture of structure materials, fission products and irradiated fuels.

The ERMINE-V campaign (1976-1980) used four core configurations to get the integral capture cross sections of fission products and irradiated samples thanks to the oscillation technique [8] and irradiation measurements. In this paper we are focusing on two particular cores, ZONA1 and ZONA3.

The fast zones were limited to the central Aluminum ring of $35 \mathrm{~cm}$ radius where the fissile fuels were 36 inches high (approximatively $92 \mathrm{~cm}$ ). MTR fuels were used in the thermal driver zone and made with graphite and U-Al alloy with very high enriched Uranium. Those fuels were surrounded by graphite and water.

\subsection{ZONA1}

The ZONA1 core is based on the following cell (Fig. 1):

As the availability of the MOx was limited, these cells occupied a central zone and were completed with equivalent Uranium cells where $\mathrm{UO}_{2}$ enriched in ${ }^{235} \mathrm{U}$ at $27 \%$ replaced the MOx fuel. This zone was extended to a radius of $22 \mathrm{~cm}$. The adaptation zone was made from the center to the driver zone by a medium enriched uranium filter made with 3 natural Uranium plates for $127 \%$ enriched Uranium, then a natural Uranium filter, a filter made with depleted uranium and finally enriched Uranium oxide $(3.5 \%)$. A representation of this core is shown in Fig. 2:

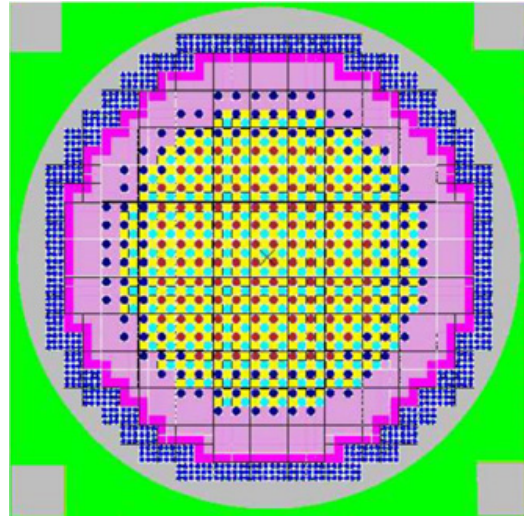

Figure 3. ZONA3 central zone.

\subsection{ZONA3}

The ZONA 3 cells are very close to the ZONA1 ones. They are more symmetrical: 8 sodium modules, $4 \mathrm{PuO}_{2}$ and 4 natural $\mathrm{UO}_{2}$. The transition zone is made by three filters and one convertor. Beyond the ZONA3 cells, equivalent $7 \%-\mathrm{UO}_{2}$ cells are made like in ZONA1 to enlarge the fast zone and then, three filters: similar $7 \%-\mathrm{UO}_{2}$ cells using depleted $\mathrm{UO}_{2}$ instead of natural $\mathrm{UO}_{2}$, depleted $\mathrm{UO}_{2}$ plates only and depleted metal Uranium. The convertor is made of $3.5 \%$ enriched $\mathrm{UO}_{2}$. A representation of this core is shown below (Fig. 3):

\section{Quantitative characterization of ZONA1 and ZONA3 cores by Monte-Carlo simulations}

\subsection{TRIPOLI-4®and JEFF 3-1-1}

Calculation capacities and nuclear data libraries have been greatly improved since the 1980's. Monte-Carlo calculations with continuous cross-sections in energy and angle and a quasi exact geometry description in 3D are now possible for such cores. ZONA1 and ZONA3 have been calculated thanks to JEFF 3.1.1 European nuclear data library [9], and TRIPOLI-4R, which is a MonteCarlo code developed at the CEA [10].

Thanks to the several documents including [5], operation books and personal communications, we were able to model the actual loading and to simulate both cores. The forward and adjoint spectra in the centers of the cores will be presented in the following sub-sections in order to evaluate the quality of the spectral adaptation.

\subsection{Simulation of ZONA1}

The first element is the effective multiplication factor: thanks to the obtained information, the reactivity of the core is less than $100 \mathrm{pcm}$. This tiny difference confirms the collected information about the loaded core (compositions of fuels, quantities, dimensions of the reflector).

Afterwards, the spectral adaptation is evaluated thanks to a comparison to the characteristics obtained in the center of a critical configuration entirely made of ZONA1cells, called reference configuration. This configuration is as high as the ZONA1 coupled core (36 inches) but its radius is far larger ( $\sim 1 \mathrm{~m}$ to be critical). The forward and adjoint spectra as well as some characteristic spectral 


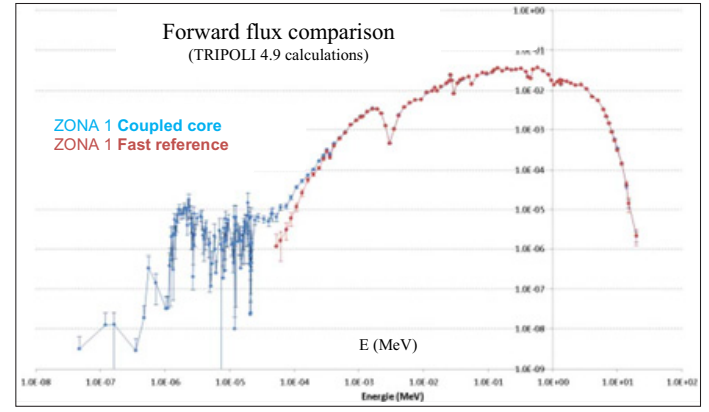

Figure 4. Forward flux - Center of ZONA1.

Table 1. Spectral indices in ZONA1 compared to the fast reference.

\begin{tabular}{|c|c|c|}
\hline ZONA 1 & Relative difference in $\%$ & $\sigma$ in $\%$ \\
\hline $\bar{\sigma}_{f}^{238} U / \bar{\sigma}_{f}^{235} U$ & -0.628 & 0.23 \\
\hline $\bar{\sigma}_{f}^{237} N p / \bar{\sigma}_{f}^{235} U$ & -0.364 & 0.18 \\
\hline $\bar{\sigma}_{C}^{238} U / \bar{\sigma}_{f}^{235} U$ & -0.792 & 0.18 \\
\hline $\bar{\sigma}_{f}^{239} \mathrm{Pu} / \bar{\sigma}_{f}^{235} U$ & -0.095 & 0.15 \\
\hline
\end{tabular}

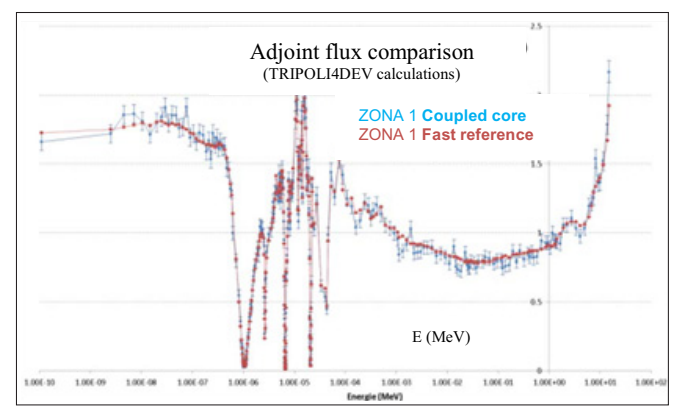

Figure 5. Adjoint flux - Center of ZONA1.

Table 2. Spectral indices in ZONA3 compared to the fast reference.

\begin{tabular}{|l|c|c|}
\hline ZONA 3 & Relative difference in \% & $\sigma$ in \% \\
\hline $\bar{\sigma}_{f}^{238} \mathrm{U} / \bar{\sigma}_{f}^{235} \mathrm{U}$ & -3.26 & 0.13 \\
\hline $\bar{\sigma}_{f}^{237} \mathrm{~Np} / \bar{\sigma}_{f}^{235} \mathrm{U}$ & -0.99 & 0.10 \\
\hline $\bar{\sigma}_{C}^{238} \mathrm{U} / \bar{\sigma}_{f}^{235} \mathrm{U}$ & -1.05 & 0.10 \\
\hline $\bar{\sigma}_{f}^{239} \mathrm{Pu} / \bar{\sigma}_{f}^{235} \mathrm{U}$ & 0.74 & 0.10 \\
\hline
\end{tabular}

indices are evaluated in a central fictive ZONA1 cell, with $16 \mathrm{~cm}$ height. The same evaluation is done in the reference configuration. The forward spectrum is shown on Fig. 4:

The adaptation of the forward flux is excellent in the fast zone $(\mathrm{E}>100 \mathrm{eV})$, while a small slowing down contribution can be observed between $1 \mathrm{eV}$ and $100 \mathrm{eV}$ (epi-thermal zone). Below $1 \mathrm{eV}$, the difference is negligible and mainly due to statistical biases. These conclusions are confirmed by the comparisons of the spectral indices (Table 1):

The adjoint spectrum calculated with TRIPOLI4-DEV using the IFP technique is also very well reproduced, even in the resolved resonance range and especially the main resonances of Plutonium isotopes:

\subsection{Simulation of ZONA3}

The same process is realized with the ZONA3 core. Its performances are evaluated through the comparisons of spectral indices (Table 2) and adjoint flux profiles (Fig. 6:

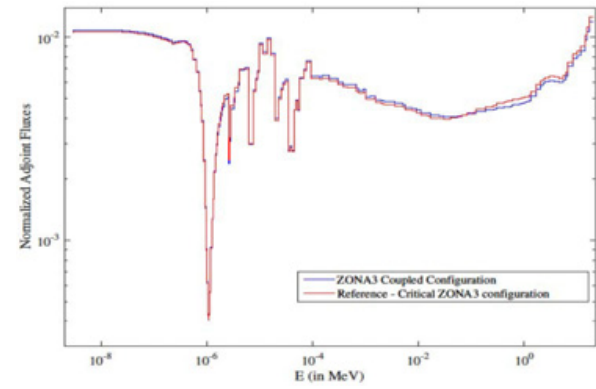

Figure 6. Adjoint flux - Center of ZONA3.

The adjoint flux is calculated by ERANOS. Both forward and adjoint fluxes are quite well reproduced even though the results are less representative than in the ZONA1 core.

\section{Re-interpretation of separated fission product oscillations}

Several samples of fission products (powders) were oscillated in the centers of ZONA1 and ZONA3 cores. This insertion of capturing materials induces a small decrease of reactivity, automatically compensated by an external rotor in $\mathrm{Cd}$ (automated pilot rod) in order to keep the reactor in its critical state [8]. The measured reactivity effect is mainly linked to the capture cross section of the inserted isotope. In order to precisely evaluate the cross sections, reactivity effects are presented by mass unit and relatively to the reactivity effect of ${ }^{235} \mathrm{U}$. It has been checked that the reactivity effect of ${ }^{235} \mathrm{U}$ is linear with mass.

The ${ }^{99} \mathrm{Tc},{ }^{103} \mathrm{Rh},{ }^{133} \mathrm{Cs}$ and ${ }^{141} \mathrm{Pr}$ isotopes were oscillated. Only ${ }^{99} \mathrm{Tc}$ and ${ }^{103} \mathrm{Rh}$ were pure whereas ${ }^{133} \mathrm{Cs}$ was linked to $\mathrm{Cl}$ and ${ }^{141} \mathrm{Pr}$ to $\mathrm{O}$ through $\operatorname{Pr}_{6} \mathrm{O}_{1} 1$. The experimental uncertainty associated to the ${ }^{141} \mathrm{Pr}$ measurement is large and then the results on this isotope will not be exploited here.

To evaluate small reactivity effect (from 0.5 to $5 \mathrm{pcm}$ ) with precision, classical Monte-Carlo techniques are not efficient because of the statistical uncertainties resulting in the difference of two eigenvalue computations. In this work, we use the IFP technique, newly implemented in TRIPOLI4-DEV [11].

Concerning ${ }^{99} \mathrm{Tc},{ }^{103} \mathrm{Rh},{ }^{133} \mathrm{Cs}$, the results obtained in ZONA1 core are resumed in Table 3:

The combined improvement of calculation tools and associated cross-section library allows a real improvement of the calculated reactivity effects: the $\mathrm{C} / \mathrm{E}-1$ ratios are closer to 0 and the uncertainties are significantly reduced in the three cases. However, the origin of those reductions can't be discriminated between the calculation capacities and descriptions or a better knowledge of nuclear data. Additional calculations will be performed using other ND libraries to investigate the source of improvement.

The $\mathrm{C} / \mathrm{E}-1$ ratios are included in the $3 \sigma$ range for both ${ }^{133} \mathrm{Cs}$ and ${ }^{99} \mathrm{Tc}$ whereas it is not for ${ }^{103} \mathrm{Rh}$. A deeper work should be done to investigate technological uncertainties, especially on MOx fuels. Then, it would be possible to determine if the difference should lead to a new evaluation of the capture cross-section of this element.

A future publication will present re-interpretation of ZONA3 oscillation measurements. 
Table 3. Oscillation measurements in ZONA1.

\begin{tabular}{|c|c|c|c|c|}
\hline \multicolumn{2}{|c|}{ ZONA1 } & ${ }^{99} \mathrm{Tc}$ & ${ }^{103} \mathrm{Rh}$ & ${ }^{133} \mathrm{Cs}$ \\
\hline \multicolumn{2}{|c|}{$\begin{array}{l}\delta \mathbf{k}(\mathbf{P F}) / \delta \mathbf{k}(\mathrm{U} 5) \\
\text { experimental }\end{array}$} & $\begin{array}{l}-0.45 \\
\pm 0.02\end{array}$ & $\begin{array}{l}-0.481 \\
\pm 0.005\end{array}$ & $\begin{array}{l}-0.30 \\
\pm 0.02\end{array}$ \\
\hline \multicolumn{2}{|c|}{$\begin{array}{l}\delta \mathbf{k}(\mathbf{P F}) / \delta \mathbf{k}(\mathbf{U} 5) \\
\text { calculated }(2016)\end{array}$} & $\begin{array}{l}-0.5122 \\
\pm 0.003\end{array}$ & $\begin{array}{l}-0.4544 \\
\pm 0.004\end{array}$ & $\begin{array}{l}-0.3034 \\
\pm 0.039\end{array}$ \\
\hline $\mathrm{C} / \mathrm{E}-1$ in $\%$ & $\begin{array}{l}1980 \\
2016\end{array}$ & $\begin{array}{l}46 \pm 9 \\
13.8 \pm 5.1\end{array}$ & $\begin{array}{l}17 \pm 5 \\
-5.5 \pm 1.4\end{array}$ & $\begin{array}{l}23 \pm 12 \\
-1.2 \pm 13\end{array}$ \\
\hline
\end{tabular}

\section{Conclusions and perspectives}

This work demonstrates the pertinence of fast-thermal coupled configurations to measure integral cross-sections in the fast energy range with great accuracy (precisions of $0.01 \mathrm{pcm}$ can be reached thanks to the oscillation technique in MINERVE). The MINERVE facility, together with its EOLE neighbor, will close by the end of 2017 and will be replaced by the multipurpose ZPR ZEPHYR by 2025 [12], whose particular goal (among others) is to better reproduce the fast energy range for ND improvement, based on spectral emphasis. In this perspective, dedicated design studies on fast-thermal coupled cores have been recently initiated because the driver zone with be thermal. It aims to get a better general understanding of these configurations and complete the demonstration of their performances [13].

Some exploratory techniques have been developed for 50 years to split the reactivity effects in their main components, to get information on absorption, fission or scattering cross-sections. It includes variations of the adjoint spectrum energy profile in order to cancel the scattering effects or to increase them thanks to the spectral emphasis method [7-13]. Moreover, by adding a local measurement of the flux depression during the oscillation phase, two different information, called local and global signals, are then available and can lead to a separation of fission and capture, or scattering and capture of the reactivity effect for fissile or inert samples respectively [14].

\section{References}

[1] S. Cathalau et al. "More than forty years of neutronics experiments in critical facilities of Cadarache center: from EOLE and MINERVE to the future ZEPHYR reactor" IGORR conference, Bariloche (2014)

[2] G. Palmiotti, M. Salvatores "Impact of Nuclear Data Uncertainties on Innovative Fast Reactors and Required Target Accuracies" Journal of Nuclear Science and Technology, 48(4), 612-619 (2011)
[3] E. Privas “Contribution à l'évaluation des incertitudes sur les sections efficaces neutroniques, pour les réacteurs à neutrons rapides", $\mathrm{PhD}$ Thesis, Université de Grenoble (2015)

[4] F. Varaine et al. "Pre-conceptual design study of ASTRID core" ICAPP Conference (2012)

[5] L. Martin-Deidier "Mesure intégrale de la capture des produits de fission dans les réacteurs à neutrons rapides" $\mathrm{PhD}$ Thesis, Université d'Orsay (1979)

[6] M. Pesic "Coupled fast-thermal system at the "RB" Nuclear reactor" Kernenergie 80, 4 (1987)

[7] K. Fahrmann et al. "A fast-thermal coupled system with energy-independent adjoint flux" Kernenergie 24, 431-433 (1981)

[8] W. Foell "Small sample reactivity measurements in nuclear reactors" American Nuclear Society \& U.S. Atomic Energy Commission (1972)

[9] A. Santamarina et al. The JEFF-3.1.1 Nuclear Data Library. NEA 2009

[10] E. Brun et al. "TRIPOLI-4®, CEA, EDF and AREVA reference Monte Carlo code" Annals of Nuclear Energy 82, 151-160 (2015)

[11] G. Truchet et al. "Continuous-Energy Adjoint Flux and Perturbation Calculation using the Iterated Fission Probability Method in Monte Carlo Code TRIPOLI-4 and Underlying Applications" SNA + MC 2013, 03504 (2014)

[12] P. Blaise et al. "Experimental R\&D innovation for GEN-II, III \& IV neutronics studies in ZPRs: a path to the future ZEPHYR facility in Cadarache" IGORR Conference, Berlin (2016)

[13] P. Ros et al. "Fast-thermal coupled cores in ZPR revisited: physical specificities and potentialities for ZEPHYR" PHYSOR Conference, Sun Valley (2016)

[14] B. Laponche et al. "Oscillation if fissile materials in thermal neutron critical assemblies: the equivalent sample method" Nuclear Science and Engineering 48, 305-318 (1972) 